\title{
Calculation of the creation and annihilation operators of a free particle in the
} light cone coordinate formalism

\section{Cálculo dos operadores de criação e aniquilação de uma partícula livre no}

\section{formalismo das coordenadas do cone luz}

Article Info:

Article history: Received 2021-09-02 / Accepted 2021-10-18 / Available online 2021-10-19

doi: $10.18540 /$ jcecvl7iss4pp13317-01-15e

Jorge Kysnney Santos Kamassury

ORCID: https://orcid.org/0000-0001-8335-9796

Federal University of Santa Catarina, Brazil

E-mail: jorge.kamassury@posgrad.ufsc.br

Damião Pedro Meira Filho

ORCID: https://orcid.org/0000-0003-0421-1094

Federal Institute of Education, Science and Technology of Pará, Brazil

E-mail: damiao.meira@ifpa.edu.br

Charles da Rocha Silva

ORCID: https://orcid.org/0000-0003-4034-8813

Federal Institute of Education, Science and Technology of Pará, Brazil

E-mail: charles.rocha@ifpa.edu.br

João Bosco Soares Pampolha Júnior

ORCID: https://orcid.org/0000-0002-6620-1847

Federal Institute of Education, Science and Technology of Pará, Brazil

E-mail: joao.pampolha@,ifpa.edu.br

Robhyson Denys Rodrigues da Silva

ORCID: https://orcid.org/0000-0002-4745-6473

Federal Institute of Education, Science and Technology of Pará, Brazil

E-mail: robhyson.denys@ifpa.edu.br

Asael Ribeiro Pinto

ORCID: https://orcid.org/0000-0002-6115-1684

Federal Institute of Education, Science and Technology of Pará, Brazil

E-mail: asael.pinto@ifpa.edu.br

\section{Resumo}

Este trabalho apresenta didaticamente os trâmites matemáticos exigidos para a construção dos operadores de criação e aniquilação para uma partícula quântica livre considerando as coordenadas do cone de luz. Para tanto, são elencadas as relações entre as coordenadas supracitadas e as coordenadas ( $c t, x, y, z$ ), além do uso da equação de Klein-Gordon-Fock no formalismo das coordenadas do cone de luz. Por fim, obtêm-se o operador evolução temporal e os operadores quânticos de criação e de aniquilação do tipo integral de movimento.

Palavras-chave: Coordenadas do cone de luz. Operadores quânticos. Operador de criação. Operador de aniquilação. Equação de Klein-Gordon-Fock.

\section{Abstract}

This work didactically presents the mathematical procedures required for the construction of the creation and annihilation operators for a free quantum particle considering the coordinates of the light cone. For that, the relationships between the aforementioned coordinates and the coordinates 
$(c t, x, y, z)$ are listed, in addition to the use of the Klein-Gordon-Fock equation in the formalism of the light cone coordinates. Finally, the temporal evolution operator and the quantum operators of creation and annihilation of the integral type of motion are obtained.

Keywords: Coordinates of the cone of light. Quantum Operators. Creation operator. Annihilation operator. Klein-Gordon-Fock equation.

\section{Introduction}

Originally introduced by the English physicist Paul Dirac, the light cone coordinates (also called the light front coordinates) were presented with the aim of constructing ways to describe the relativistic dynamics of a physical system (Dirac, 1949). In short, the original idea consists of combining the principles of special relativity with the Hamiltonian formulation so that the only requirement is that the theory satisfy the properties of the Poincare group.

Due to its properties, in recent decades, the light cone coordinates have been used, for example, in studies of quantum field theory and quantum chromodynamics under low energy regime. Specifically in the context of the present work, these coordinates enable the algebraic structure of the Klein-Gordon-Fock relativistic equation (abbreviated, KGFE). Obtained in 1926, independently by physicists Oskar Klein, Walter Gordon and Vladimir Fock, the KGFE (also called the Klein-Fock-Gordon equation or just the Klein-Gordon equation) is historically recognized as the first relativistic wave equation, having applications in condensed matter physics and dispersive wave phenomena, in addition to allowing the description of the behavior of particles with zero spin (Ohlsson, 2011; Meira Filho \& Kamassury, 2018; Veeresha et al., 2020).

Also within the scope of KGFE, as identified by Bagrov et al. (1976), by enabling the

exchange of the second-order partial differential in time $\left(\frac{\partial}{\partial t^{2}}\right)$ for a first-order partial differential in the variable $\left(\frac{\partial}{\partial u^{0}}\right)$, the light cone coordinate system facilitates the construction of the temporal evolution operator from which it is possible to establish a new annihilation operator with motion integral status. In the context of semiclassical quantum states, in a relativistic regime, this new operator is suitable for obtaining coherent quantum states of a charged quantum particle under the influence of different classical electromagnetic field configurations.

In practice, this algebraic facilitation has its appeal in supporting theoretical studies of coherent states so useful in modern quantum theory, with immediate applications in quantum field theory, loop quantum gravity, and quantum computation (Pereira \& Miranda, 2002; Gazeau, 2009; Bagrov et al., 2015).

That said, under a primarily pedagogical scope, this work presents the stages of algebraic development for the construction of the creation and annihilation operators for a free particle under the formalism of light coordinates. Therefore, initially, the relationships between the light cone coordinates and the Minkowski space-time coordinates are listed. Subsequently, the KGFE is presented using the generalized curvilinear coordinates $u^{\mu}$. Finally, in the last two sections, the temporal evolution operator and the integral motion type operator are obtained.

\section{Minkowski space coordinates and light cone coordinates}

Briefly, to identify associations between Minkowski spacetime coordinates and the light cone coordinates, consider the diagram in Figure 1 inspired by the approach presented by Kamassury et al. (2020), in which, $(d s)^{2}=c^{2}(d t)^{2}-(d z)^{2}$ is the line element of a two-dimensional subspace 
( $z$ and $c t$ ). Relating the coordinates of the cone of light $\left(u^{0}\right.$ and $\left.u^{3}\right)$ to the coordinates $\left(z\right.$ and $x^{0}=$ $c t$ ), we achieve:

$$
\begin{aligned}
& u^{0}=+z \cos (45)+x^{0} \operatorname{sen}(45)=\frac{x^{0}+z}{\sqrt{2}} \\
& u^{3}=-z \cos (45)+x^{0} \operatorname{sen}(45)=\frac{x^{0}-z}{\sqrt{2}}
\end{aligned}
$$
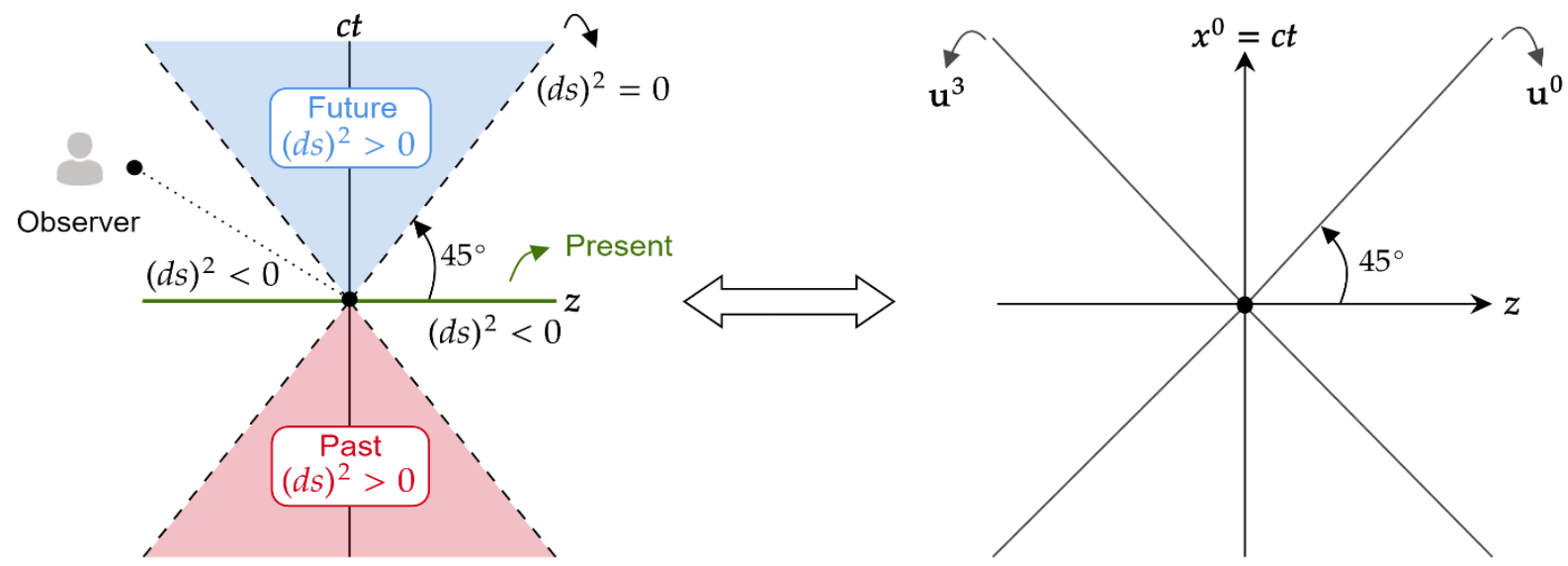

Figure 1 - Diagram and coordinates of the cone of light.

In possession of Equations 1 and 2 and generalizing to four-dimensional space, we obtain both Minkowski space-time coordinates.

$$
x^{\mu}=\left(x^{0}, x^{1}, x^{2}, x^{3}\right)=(c t, x, y, z)
$$

as well as the coordinates of the cone of light:

$$
u^{\mu}=\left(u^{0}, u^{1}, u^{2}, u^{3}\right)=\left(\frac{x^{0}+z}{\sqrt{2}}, x, y, \frac{x^{0}-z}{\sqrt{2}}\right)=\left(\frac{c t+z}{\sqrt{2}}, x, y, \frac{c t-z}{\sqrt{2}}\right)
$$

\section{Klein-Gordon-Fock equation for a free particle in light cone coordinates}

Considering the generalized curvilinear space-time coordinates $u^{\mu}$ in terms of Cartesian and time coordinates $x^{\mu}$, the KGFE for a free and relativistic particle can be written as

$$
\sum_{\mu=0}^{3} \sum_{\nu=0}^{3} \tilde{g}^{\mu \nu}\left(\tilde{p}_{\mu} \tilde{p}_{\nu}-i \sum_{\alpha=0}^{3} \Gamma_{\mu \nu}^{\alpha} \tilde{p}_{\alpha}\right) \Psi=m_{0}^{2} c^{2} \Psi
$$

where the metric tensor $\tilde{g}^{\mu \nu}$, the symbol of Christoffel $\Gamma_{\alpha \beta}^{\mu}$ and the term $\tilde{p}_{\mu}$ são, are, respectively:

$$
\tilde{g}^{\mu \nu}=\sum_{\alpha=0}^{3} \sum_{\beta=0}^{3} \frac{\partial u^{\mu}}{\partial x^{\alpha}} \frac{\partial u^{v}}{\partial x^{\beta}} \eta^{\alpha \beta}
$$




$$
\begin{aligned}
& \Gamma_{\alpha \beta}^{\mu}=\frac{1}{2} \sum_{\nu=0}^{3} \tilde{g}_{\mu \nu}\left(\partial_{\alpha} \tilde{g}_{v \beta}+\partial_{\beta} \tilde{g}_{v \alpha}-\partial_{\nu} \tilde{g}_{\alpha \beta}\right) \\
& \tilde{p}_{\mu}=i \hbar \frac{\partial}{\partial u^{\mu}}
\end{aligned}
$$

Using the relationships expressed in Equation 4 and knowing that the non-null components of the metric $\eta^{\mu \nu}$ are $\eta^{j j}=1$ for $j=0,1,2,3$, we have:

$$
\begin{aligned}
& g^{00}=g^{01}=g^{02}=g^{10}=g^{12}=g^{13}=g^{20} g^{21}=g^{23}=g^{31}=g^{32}=g^{33}=0 \\
& g^{03}=-g^{11}=-g^{22}=g^{30}=1
\end{aligned}
$$

Observing these last relationships, it is concluded that the components of the Christoffel symbol are all null $\left(\Gamma_{\alpha \beta}^{\mu}=0\right)$, which allows rewriting Equation 5 as:

$$
\sum_{\mu=0}^{3} \sum_{\nu=0}^{3} \tilde{g}^{\mu v} \tilde{p}_{\mu} \tilde{p}_{\nu} \Psi=m_{0}^{2} c^{2} \Psi
$$

For the next steps, let us consider the relationships between the coordinates $(c t, x, y, z)$ and $\left(u^{0}, u^{1}, u^{2}, u^{3}\right)$ of the differential operators shown in Table 1 . That said, we can adequately substitute the relations of the partial differential operators in Equation 11 as follows

$$
\begin{gathered}
\frac{1}{2}\left(\frac{\partial}{\partial u^{0^{2}}}+2 \frac{\partial^{2}}{\partial u^{0} \partial u^{3}}+\frac{\partial^{2}}{\partial u^{3^{2}}}\right) \Psi-\sum_{j=1}^{2} \frac{\partial^{2} \Psi}{\partial u^{i^{2}}}-\frac{1}{2}\left(\frac{\partial}{\partial u^{0^{2}}}-2 \frac{\partial^{2}}{\partial u^{0} \partial u^{3}}+\frac{\partial^{2}}{\partial u^{3^{2}}}\right) \Psi \\
=-\frac{m_{0} c^{2}}{\hbar^{2}} \Psi
\end{gathered}
$$

resulting in the expression:

$$
\frac{\partial \Psi}{\partial u^{0}}=\left(\frac{\partial}{\partial u^{3}}\right)^{-1}\left[\frac{1}{2}\left(\frac{\partial^{2} \Psi}{\partial u^{1^{2}}}+\frac{\partial^{2} \Psi}{\partial u^{2^{2}}}-\frac{m_{0}^{2} c^{2}}{\hbar} \Psi\right)\right]
$$

Table 1 - Relationship between the differential operators of the cartesian and time coordinate systems and the light cone coordinates.

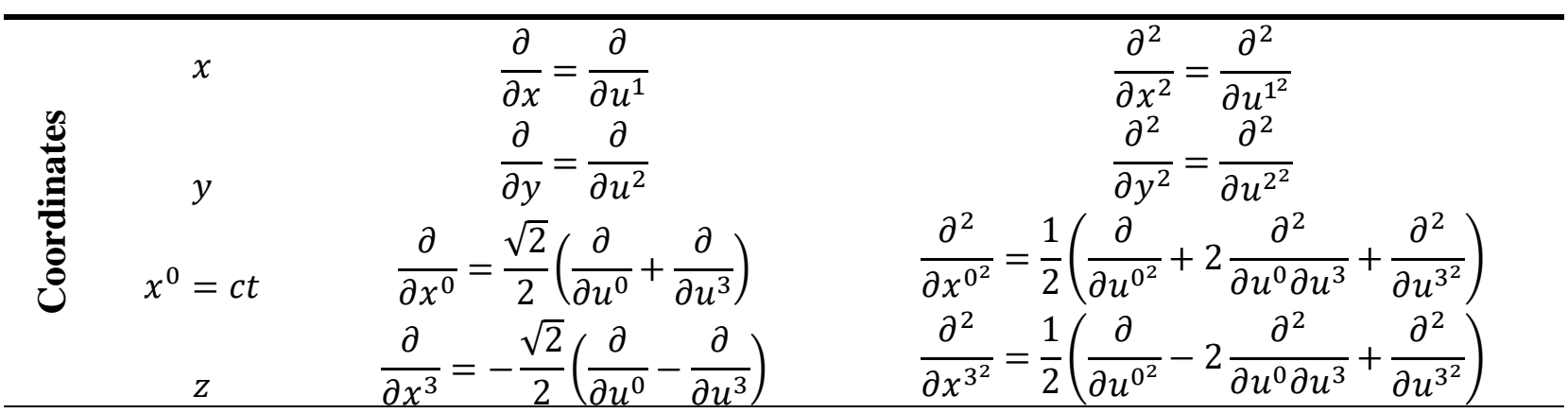


The Equation 13 is recognized as KGFE for a free particle written in terms of the coordinates of the cone of light. Alternatively, it is possible to rewrite it algebraically similar to the famous Schrödinger equation (Bagrov \& Gitman, 1990), ie

$$
\frac{\partial \Psi}{\partial u^{0}}=\widehat{\mathcal{H}} \Psi
$$

where the wave function $\Psi$ is described in terms of coordinates $\left(u^{1}, u^{2}, u^{3}, u^{0}\right)$ and the Hamiltonian type operator $\widehat{\mathcal{H}}$ is given by:

$$
\widehat{\mathcal{H}}=\frac{1}{2}\left(\frac{\partial}{\partial u^{3}}\right)^{-1}\left(\frac{\partial^{2}}{\partial u^{1^{2}}}+\frac{\partial^{2}}{\partial u^{2^{2}}}-\frac{m_{0}^{2} c^{2}}{\hbar}\right)
$$

\section{Time evolution operator}

To obtain the time evolution operator, consider an electrically charged spin-zero free quantum particle whose dynamics is described by KGFE in the light cone coordinate system expressed in Equation 13 which can be rewritten as:

$$
2 \frac{\partial^{2} \Psi}{\partial u^{0} \partial u^{3}}-\frac{\partial^{2} \Psi}{\partial u^{1^{2}}}-\frac{\partial^{2} \Psi}{\partial u^{2^{2}}}=-\frac{m_{0}^{2} c^{2}}{\hbar^{2}} \Psi
$$

Furthermore, we can define the creation $(a)$ and annihilation $\left(a^{+}\right)$operators associated with the coordinates $u^{1}$ and $u^{2}$ as:

$$
\begin{aligned}
& \hat{a}_{1}=\left(\frac{\partial}{\partial u^{1}}+i \frac{\partial}{\partial u^{2}}\right), \quad \hat{a}_{1}^{+}=\left(\frac{\partial}{\partial u^{1}}-i \frac{\partial}{\partial u^{2}}\right) \\
& \hat{a}_{2}=\left(i \frac{\partial}{\partial u^{2}}-\frac{\partial}{\partial u^{1}}\right), \hat{a}_{2}^{+}=-\left(\frac{\partial}{\partial u^{1}}+i \frac{\partial}{\partial u^{2}}\right)
\end{aligned}
$$

Then, the expression $\hat{a}_{1} \hat{a}_{1}^{+} \Psi$ is conveniently calculated as follows:

$$
\begin{aligned}
\hat{a}_{1} \hat{a}_{1}^{+} \Psi & =\left(\frac{\partial}{\partial u^{1}}+i \frac{\partial}{\partial u^{2}}\right)\left(\frac{\partial}{\partial u^{1}}-i \frac{\partial}{\partial u^{2}}\right) \Psi=\left(\frac{\partial}{\partial u^{1}}+i \frac{\partial}{\partial u^{2}}\right)\left(\frac{\partial \Psi}{\partial u^{1}}-i \frac{\partial \Psi}{\partial u^{2}}\right) \\
& =\sum_{i=1}^{2} \frac{\partial^{2} \Psi}{\partial u^{i^{2}}}-i \frac{\partial^{2} \Psi}{\partial u^{1} \partial u^{2}}+i \frac{\partial^{2} \Psi}{\partial u^{2} \partial u^{1}}
\end{aligned}
$$

And, analogously, the expression $\hat{a}_{2} \hat{a}_{2}^{+} \Psi$ :

$$
\hat{a}_{2} \hat{a}_{2}^{+} \Psi=\sum_{i=1}^{2} \frac{\partial^{2} \Psi}{\partial u^{i^{2}}}-i \frac{\partial^{2} \Psi}{\partial u^{2} \partial u^{1}}+i \frac{\partial^{2} \Psi}{\partial u^{1} \partial u^{2}}
$$

Verifying that $\frac{\partial^{2} \Psi}{\partial u^{1} \partial u^{2}}=\frac{\partial^{2} \Psi}{\partial u^{2} \partial u^{1}}$, we rewrite Equations 19 and 20, in this order, as 


$$
\hat{a}_{i} \hat{a}_{i}^{+} \Psi=\sum_{i=1}^{2} \frac{\partial^{2} \Psi}{\partial u^{i^{2}}}, \text { where } i=1,2
$$

as well as the Hamiltonian operator $\widehat{\mathcal{H}}$ present in Equation 14 in the following format:

$$
\widehat{\mathcal{H}}=\frac{1}{2}\left(\frac{\partial}{\partial u^{3}}\right)^{-1}\left(\frac{\hat{a}_{1} \hat{a}_{1}^{+}}{2}+\frac{\hat{a}_{2} \hat{a}_{2}^{+}}{2}-\frac{m_{0}^{2} c^{2}}{\hbar^{2}}\right)
$$

In the meantime, defining the operator $\widehat{\mathcal{K}}_{3}=\frac{\partial}{\partial u^{3}}$ it is essential to obtain the switch between this and the operator $\widehat{\mathcal{H}}$, with the application of this switch on the wave function $\Psi=$ $\Psi\left(u^{1}, u^{2}, u^{3}, u^{0}\right)$, this is:

$$
\left[\widehat{\mathcal{H}}, \widehat{\mathcal{K}}_{3}\right]_{-} \Psi=\widehat{\mathcal{H}} \widehat{\mathcal{K}}_{3} \Psi-\widehat{\mathcal{K}}_{3} \widehat{\mathcal{H}} \Psi
$$

By algebraically developing the switch, we obtain

$$
\left[\widehat{\mathcal{H}}, \widehat{\mathcal{K}}_{3}\right]_{-} \Psi=\frac{1}{2}\left(\widehat{\mathcal{K}}_{3}\right)^{-1}(\Gamma) \widehat{\mathcal{K}}_{3} \Psi-\widehat{\mathcal{K}}_{3} \frac{1}{2}\left(\widehat{\mathcal{K}}_{3}\right)^{-1} \Gamma \Psi
$$

wherein:

$$
\Gamma=\sum_{i=1}^{2} \frac{\hat{a}_{i} \hat{a}_{i}^{+}}{2}-\frac{m_{0}^{2} c^{2}}{\hbar^{2}}
$$

Then knowing that

$$
\widehat{\mathcal{K}}_{3} \hat{a}_{1} \hat{a}_{1}^{+} \Psi=\hat{a}_{1} \hat{a}_{1}^{+} \widehat{\mathcal{K}}_{3} \Psi
$$

and substituting this last relationship in Equation 24, we reach:

$$
\left[\widehat{\mathcal{H}}, \widehat{\mathcal{K}}_{3}\right]_{-} \Psi=\frac{1}{2}\left(\widehat{\mathcal{K}}_{3}\right)^{-1}(\Gamma) \widehat{\mathcal{K}}_{3} \Psi-\frac{1}{2}\left(\widehat{\mathcal{K}}_{3}\right)^{-1} \Gamma \mathcal{K}_{3}=0
$$

Based on the Heisenberg formalism, quantum states that describe the temporal evolution of pure quantum states that belong to Hilbert space, in addition to the observables associated with timeindependent operators $\hat{\mathcal{A}}$ can be, in Schrödinger's formalism, replaced by operators $\hat{\mathcal{A}}(t)$, which in turn evolve through a unitary transformation, namely:

$$
\hat{\mathcal{A}}(t)=\exp \left(\frac{i \widehat{\mathcal{H}} t}{\hbar}\right) \hat{\mathcal{A}} \exp \left(-\frac{i \widehat{\mathcal{H}} t}{\hbar}\right)
$$

Since the operator $\hat{\mathcal{A}}(t)$ satisfies the following equation of motion

$$
i \hbar \frac{d \hat{\mathcal{A}}(t)}{d t}=[\hat{\mathcal{A}}, \widehat{\mathcal{H}}]_{-}
$$

we can verify that: 


$$
-i \hbar \frac{d \widehat{\mathcal{K}}_{3}}{d t}=\left[\widehat{\mathcal{H}}, \widehat{\mathcal{K}}_{3}\right]_{-}=0
$$

This last result allows us to state that the operator $\widehat{\mathcal{K}}_{3}=\frac{\partial}{\partial u^{3}}$ is an integral motion type operator for Equation 13. In other terms:

$$
-i \frac{\partial}{\partial u^{3}} \Psi=\mathcal{K}_{3} \Psi
$$

Also, assuming that operator $\mathcal{K}_{3}$ is a constant, it is feasible to rewrite the wave function in the form

$$
\Psi\left(u^{1}, u^{2}, u^{3}, u^{0}\right)=\exp \left(-i \mathcal{K}_{3} u^{3}\right)
$$

such that

$$
\widehat{\mathcal{K}}_{3} \Psi\left(u^{1}, u^{2}, u^{3}, u^{0}\right)=-i \mathcal{K}_{3} \Psi\left(u^{1}, u^{2}, u^{3}, u^{0}\right)
$$

wich ensures that $\Psi$ is an eigenfunction of the operator $\widehat{\mathcal{K}}_{3}$ with corresponding eigenvalue $\mathcal{K}_{3}$.

In this way, revisiting the KGFE modeling in the format of the Schrödinger equation highlighted in Equation 14, that is,

$$
\frac{\partial \Psi\left(u^{1}, u^{2}, u^{3}, u^{0}\right)}{\partial u^{0}}=\widehat{\mathcal{H}} \Psi\left(u^{1}, u^{2}, u^{3}, u^{0}\right)
$$

performing the proper replacement of the wave function $\Psi$ by Equation 32

$$
\frac{\partial}{\partial u^{0}} \exp \left[-i \mathcal{K}_{3} u^{3} \Psi\right]=\frac{1}{2}\left(\frac{\partial}{\partial u^{3}}\right)^{-1}\left(\hat{\Xi}-\frac{m_{0}^{2} c^{2}}{\hbar^{2}}\right) \exp \left(-i \mathcal{K}_{3} u^{3}\right) \Psi
$$

and developing it algebraically

$$
\frac{\partial}{\partial u^{3}} \exp \left(-i \mathcal{K}_{3} u^{3}\right) \frac{\partial \Psi}{\partial u^{0}}=\left(\widehat{\Xi}-\frac{m_{0}^{2} c^{2}}{\hbar^{2}}\right) \exp \left(-i \mathcal{K}_{3} u^{3}\right) \Psi
$$

we get

$$
\frac{\partial \Psi}{\partial u^{0}}=-\frac{1}{2 i \mathcal{K}_{3}}\left(\hat{\Xi}-\frac{m_{0}^{2} c^{2}}{\hbar^{2}}\right) \Psi
$$

where $\widehat{\Xi}=\sum_{j=1}^{2} \frac{\partial^{2}}{\partial u^{j^{2}}}$. Then redefining $\Psi$ and $\widehat{\mathcal{H}}$ to

$$
\begin{aligned}
\Psi & =\widehat{U}\left(u^{0}\right) \Psi\left(u^{1}, u^{2}\right) \\
\widehat{\mathcal{H}} & =-\frac{1}{2 i \mathcal{K}_{3}}\left(\sum_{i=1}^{2} \frac{\partial^{2} \Psi}{\partial u^{i^{2}}}-\frac{m_{0}^{2} c^{2}}{\hbar^{2}}\right)
\end{aligned}
$$

rewrite Equation (37) as follows: 


$$
\Psi\left(u^{1}, u^{2}\right) \frac{d \widehat{U}\left(u^{0}\right)}{d u^{0}}=\widehat{\mathcal{U}}\left(u^{0}\right)\left[-\frac{1}{2 i \widehat{\mathcal{K}}_{3}}\left(\widehat{\Xi}-\frac{m_{0}^{2} c^{2}}{\hbar^{2}}\right)\right] \Psi=\widehat{U}\left(u^{0}\right) \widehat{\mathcal{H}} \Psi
$$

Finally, integrating both members of the last expression

$$
\begin{aligned}
& \int\left[\widehat{\mathcal{U}}\left(u^{0}\right)\right]^{-1} d \widehat{\mathcal{U}}\left(u^{0}\right)=\int \widehat{\mathcal{H}} d u^{0} \\
& \ln \left[\widehat{\mathcal{U}}\left(u^{0}\right)\right]=\int \widehat{\mathcal{H}} d u^{0}
\end{aligned}
$$

we obtain Equation (43) which is called the temporal evolution operator.

$$
\widehat{\mathcal{U}}\left(u^{0}\right)=\exp \left(\int \widehat{\mathcal{H}} d u^{0}\right)
$$

\section{Operator of creation and annihilation of the integral type of movement}

Once the temporal evolution operator is reached, it is convenient to construct the annihilation operator that behaves as a motion integral. For that purpose, consider the following expressions:

$$
\begin{aligned}
& \hat{\mathcal{A}}_{1}\left(u^{0}\right)=\hat{u}\left(u^{0}\right) \hat{a}_{1} \hat{u}\left(u^{0}\right)^{-1} \\
& \hat{\mathcal{A}}_{2}\left(u^{0}\right)=\widehat{u}\left(u^{0}\right) \hat{a}_{2} \hat{u}\left(u^{0}\right)^{-1} \\
& \hat{\mathcal{A}}_{1}\left(u^{0}\right) \Psi\left(u^{1}, u^{2}\right)=\widehat{u}\left(u^{0}\right) \hat{a}_{1} \widehat{u}\left(u^{0}\right)^{-1} \Psi\left(u^{1}, u^{2}\right)
\end{aligned}
$$

With the proper substitution of the result of Equation (43) in Equation (46), and proceeding algebraically, we reach Equation (47).

$$
\begin{aligned}
\hat{\mathcal{A}}_{1}\left(u^{0}\right) \Psi\left(u^{1}, u^{2}\right) & =\exp \left(\int \widehat{\mathcal{H}} d u^{0}\right) \hat{a}_{1} \exp \left(\int-\widehat{\mathcal{H}} d u^{0}\right) \Psi\left(u^{1}, u^{2}\right) \\
& =\widehat{U}\left(u^{0}\right) \hat{a}_{1} \widehat{U}\left(u^{0}\right)^{-1} \Psi\left(u^{1}, u^{2}\right)
\end{aligned}
$$

Using McLaurin series for exponential functions (Stewart, 2012)

$$
\begin{aligned}
& e^{x}=\sum_{n=0}^{\infty} \frac{x^{n}}{n !}=1+x+\frac{x^{2}}{2}+\frac{x^{3}}{6}+\cdots \\
& e^{-x}=\sum_{n=0}^{\infty} \frac{(-x)^{n}}{n !}=1-x+\frac{x^{2}}{2}-\frac{x^{3}}{6}+\cdots
\end{aligned}
$$

adopting conveniently $\widehat{w}=\int \frac{i}{2 \mathcal{K}_{3}} \widehat{\Xi} d u^{0}$ and performing some algebraic steps, we resulted in:

$$
\begin{aligned}
e^{\widehat{w}} \hat{a}_{1} e^{-\widehat{w}} & =\left(1+\widehat{w}+\frac{\widehat{w}^{2}}{2}+\cdots\right) \hat{a}_{1}\left(1-\widehat{w}+\frac{\widehat{w}^{2}}{2}+\cdots\right) \\
& =\hat{a}_{1}+\left[\widehat{w}, \hat{a}_{1}\right]+\frac{1}{2}\left(\widehat{w}^{2} \hat{a}_{1}-2 \widehat{w} \hat{a}_{1} \widehat{w}+\widehat{w}^{2} \hat{a}_{1}\right)+\cdots
\end{aligned}
$$

Rewriting the term in parentheses from Equation 51 


$$
\begin{aligned}
\widehat{w}^{2} \hat{a}_{1}-2 \widehat{w} \hat{a}_{1} \widehat{w}+\widehat{w}^{2} \hat{a}_{1} & =\widehat{w}\left(\widehat{w} \hat{a}_{1}-\hat{a}_{1} \widehat{w}\right)-\left(\widehat{w} \hat{a}_{1}-\hat{a}_{1} \widehat{w}\right) \widehat{w} \\
& =\widehat{w}\left[\widehat{w}, \hat{a}_{1}\right]_{-}-\left[\widehat{w}, \hat{a}_{1}\right]_{-} \widehat{w}
\end{aligned}
$$

easily, the following expression is obtained:

$$
e^{\widehat{w}} \hat{a}_{1} e^{-\widehat{w}}=\hat{a}_{1}+\left[\widehat{w}, \hat{a}_{1}\right]_{-}+\frac{1}{2}\left[\widehat{w},\left[\widehat{w}, \hat{a}_{1}\right]_{-}\right]_{-}
$$

By carefully analyzing each term, we have:

$$
\begin{aligned}
{\left[\widehat{w}, \hat{a}_{1}\right]_{-} \Psi\left(u^{1}, u^{2}\right) } & =\left(\widehat{w} \hat{a}_{1}-\hat{a}_{1} \widehat{w}\right) \Psi\left(u^{1}, u^{2}\right) \\
& =\widehat{w} \hat{a}_{1} \Psi\left(u^{1}, u^{2}\right)-\widehat{a}_{1} \widehat{w} \Psi\left(u^{1}, u^{2}\right)
\end{aligned}
$$

Specifically for the term $\widehat{w} \hat{a}_{1} \Psi\left(u^{1}, u^{2}\right)$, is the relationship given in the Equation 54 .

$$
\begin{aligned}
\widehat{w} \hat{a}_{1} \Psi\left(u^{1}, u^{2}\right) & =\left(\int-\frac{i}{2 \mathcal{K}_{3}} \widehat{\Xi} d u^{0}\right)\left(\frac{\partial}{\partial u^{1}}+i \frac{\partial}{\partial u^{2}}\right) \Psi\left(u^{1}, u^{2}\right) \\
& =\left[\int \frac{i}{2 \mathcal{K}_{3}}\left(\frac{\partial^{2}}{\partial u^{1^{2}}}+i \frac{\partial^{2}}{\partial u^{2^{2}}}\right) d u^{0}\right]\left[\frac{\partial \Psi\left(u^{1}, u^{2}\right)}{\partial u^{1}}+i \frac{\partial \Psi\left(u^{1}, u^{2}\right)}{\partial u^{2}}\right] \\
& =\int \frac{i}{2 \mathcal{K}_{3}}\left(\frac{\partial}{\partial u^{1}}+i \frac{\partial}{\partial u^{2}}\right)\left[\frac{\partial^{2} \Psi\left(u^{1}, u^{2}\right)}{\partial u^{1^{2}}}+i \frac{\partial^{2} \Psi\left(u^{1}, u^{2}\right)}{\partial u^{2^{2}}}\right] d u^{0} \\
& =\hat{a}_{1} \widehat{w} \Psi\left(u^{1}, u^{2}\right) d u^{0}
\end{aligned}
$$

With this result in hand, it is possible to reformulate Equation 53 as

$\left[\widehat{w}, \hat{a}_{1}\right]_{-} \Psi\left(u^{1}, u^{2}\right)=0$

and consequently:

$$
e^{\widehat{w}} \hat{a}_{1} e^{-\widehat{w}} \Psi\left(u^{1}, u^{2}\right)=\hat{a}_{1} \Psi\left(u^{1}, u^{2}\right)
$$

In this panorama, we observe that

$$
\begin{aligned}
\hat{\mathcal{A}}_{1}\left(u^{0}\right) \Psi\left(u^{1}, u^{2}\right) & =\widehat{u}\left(u^{0}\right) \hat{a}_{1} \widehat{\mathcal{u}}\left(u^{0}\right)^{-1} \Psi\left(u^{1}, u^{2}\right) \\
& =\hat{a}_{1} \Psi\left(u^{1}, u^{2}\right)
\end{aligned}
$$

of which, we conclude that:

$$
\hat{\mathcal{A}}_{1}=\hat{a}_{1} \quad, \quad \hat{\mathcal{A}}_{1}^{+}=\hat{a}_{1}^{+}
$$

Using procedures similar to those described for obtaining the previous operative relationships, we verified that

$$
\hat{\mathcal{A}}_{2}=\hat{a}_{2} \quad, \quad \hat{\mathcal{A}}_{2}^{+}=\hat{a}_{2}^{+}
$$

where $\hat{\mathcal{A}}_{1}^{+}$and $\hat{\mathcal{A}}_{2}^{+}$are the adjoint operators of the operators $\hat{\mathcal{A}}_{1}$ and $\hat{\mathcal{A}}_{2}$, in this order. From Equations 17 and 18, we identified:

$$
\hat{a}_{1}=-\hat{a}_{2}^{+} \quad, \quad \hat{a}_{2}=-\hat{a}_{1}^{+}
$$


Finally, we easily reach relations analogous to Equations 44 and 45 for the operators $\hat{\mathcal{A}}_{1}^{+}$and $\hat{\mathcal{A}}_{2}^{+}$, namely

$$
\hat{\mathcal{A}}_{1}^{+}=\left[\hat{\mathrm{u}} \hat{a}_{1} \hat{u}^{-1}\right]^{+}=\left[\hat{a}_{1} \hat{u}^{-1}\right]^{+} \hat{u}^{+}=\left[\hat{u}^{-1}\right]^{+} \hat{a}_{1}^{+} \hat{u}^{+}
$$

and

$$
\hat{\mathcal{A}}_{2}^{+}=\left[\hat{\mathrm{u}} \hat{a}_{2} \hat{u}^{-1}\right]^{+}=\left[\hat{\mathcal{u}}^{-1}\right]^{+} \hat{a}_{2}^{+} \hat{\mathcal{U}}^{+}
$$

Knowing that the temporal evolution operator $\hat{\mathcal{U}}$, according to Equations 39 and 43, it is given by

$$
\widehat{u}=\exp \left[\int \frac{(-i)}{2 \widehat{\mathcal{K}}_{3}}\left(\Xi-\frac{m_{0}^{2} c^{2}}{\hbar^{2}}\right) d u^{0}\right]
$$

e can also write the inverse operator as well as the adjunct operator of the operator $\hat{u}$, in this order, as

$$
\begin{aligned}
& \widehat{u}^{-1}=\exp \left[\int \frac{i}{2 \widehat{\mathcal{K}}_{3}}\left(\frac{\partial^{2}}{\partial u^{1^{2}}}+\frac{\partial^{2}}{\partial u^{2}}-\frac{m_{0}^{2} c^{2}}{\hbar^{2}}\right) d u^{0}\right] \\
& \hat{u}^{+}=\exp \left[\int \frac{i}{2 \widehat{\mathcal{K}}_{3}}\left(\frac{\partial^{2}}{\partial u^{1^{2}}}+\frac{\partial^{2}}{\partial u^{2^{2}}}-\frac{m_{0}^{2} c^{2}}{\hbar^{2}}\right) d u^{0}\right]
\end{aligned}
$$

such that

$$
\widehat{u}^{-1}=\hat{u}^{+} \quad, \quad\left[\hat{u}^{-1}\right]^{+}=\widehat{u}
$$

and motion integral type annihilation operators are expressed as:

$$
\hat{\mathcal{A}}^{-1}=\hat{\mathcal{U}} \hat{a}_{1}^{+} \hat{\mathcal{U}}^{-1}, \hat{\mathcal{A}}_{2}^{+}=\hat{\mathcal{U}} \hat{a}_{2}^{+} \hat{U}^{-1}
$$

\section{Algebraic relations between operators $a_{1}$ and $a_{2}$}

In this section, we briefly present the algebraic relationships between the operators defined in Equations 44 and 45 which corroborate their status as motion integrals with respect to Hamiltonian given by in Equation 39. To do so, let us consider Equation 68 for the wave function $\Psi$

$$
\Psi\left(u^{0}, u^{1}, u^{2}, u^{3}\right)=\exp \left(-i \mathcal{K}_{3} u^{3}\right) \phi\left(u^{0}, u^{1}, u^{2}\right)
$$

such that:

$$
\frac{\partial}{\partial u^{0}} \Psi\left(u^{0}, u^{1}, u^{2}, u^{3}\right)=\widetilde{\widetilde{\mathcal{H}}} \Psi\left(u^{0}, u^{1}, u^{2}, u^{3}\right)
$$

Knowing that the operator of type Hamiltonian can be rewritten as below

$$
\widetilde{\mathcal{H}}=-\frac{i}{2 \hbar}\left[i \hbar \frac{\partial}{\partial u^{3}}\right]^{-1}\left[(i \hbar)^{2} \frac{\partial^{2}}{\partial u^{1^{2}}}+(i \hbar)^{2} \frac{\partial^{2}}{\partial u^{2^{2}}}+m_{0}^{2} c^{2}\right]
$$


and applying this Hamiltonian on the function given by equation 68

$$
\widetilde{\mathcal{H}}\left[\exp \left(-i \mathcal{K}_{3} u^{3}\right) \phi\left(u^{0}, u^{1}, u^{2}\right)\right]=\frac{i}{2 \mathcal{K}_{3} \hbar^{2}} \exp \left(-i \mathcal{K}_{3} u^{3}\right)\left[m_{0}^{2} c^{2} \phi+(i \hbar)^{2} \sum_{j=1}^{2} \frac{\partial^{2} \phi}{\partial u^{j^{2}}}\right]
$$

we get:

$$
\frac{\partial \phi\left(u^{0}, u^{1}, u^{2}\right)}{\partial u^{0}}=\frac{i}{2 \mathcal{K}_{3} \hbar^{2}}\left[\left(m_{0}^{2} c^{2}+(i \hbar)^{2} \sum_{j=1}^{2} \frac{\partial^{2}}{\partial u^{j^{2}}}\right) \phi\left(u^{0}, u^{1}, u^{2}\right)\right]
$$

Making use of the operator $\widehat{\mathcal{U}}$, which is presented by Equation 63, we have:

$\hat{\mathcal{A}}_{1}\left(u^{0}\right)=\hat{u}\left(u^{0}\right) \hat{a}_{1} \hat{u}\left(u^{0}\right)^{-1}=\hat{a}_{1}$

$\hat{\mathcal{A}}_{2}\left(u^{0}\right)=\hat{U}\left(u^{0}\right) \hat{a}_{2} \hat{u}\left(u^{0}\right)^{-1}=\hat{a}_{2}$

Also, it is possible to write the eigenvalue and eigenvector expressions as follows:

$\hat{\mathcal{A}}_{1} \phi=\alpha_{1} \phi=\hat{a}_{1} \phi$

$\hat{\mathcal{A}}_{2} \phi=\alpha_{2} \phi=\hat{a}_{2} \phi$

Being the function $\phi\left(u^{0}, u^{1}, u^{2}\right)$ expressed in terms of exponentials functions

$$
\phi\left(u^{0}, u^{1}, u^{2}\right)=\exp \left(a_{0} u^{0}\right) \exp \left(a_{1} u^{1}\right) \exp \left(a_{2} u^{2}\right)
$$

and applying the operators $\hat{a}_{1}$ and $\hat{a}_{2}$ on $\phi\left(u^{0}, u^{1}, u^{2}\right)$ as described below

$$
\begin{aligned}
& \left(\frac{\partial}{\partial u^{1}}+i \frac{\partial}{\partial u^{2}}\right) \phi=\alpha_{1} \phi \Rightarrow a_{1} \phi+i a_{2} \phi=\alpha_{1} \phi \Rightarrow a_{1}+i a_{2}=\alpha_{1} \\
& \left(i \frac{\partial}{\partial u^{2}}-\frac{\partial}{\partial u^{1}}\right) \phi=\alpha_{2} \phi \Rightarrow i a_{2} \phi-i a_{1} \phi=\alpha_{2} \phi \Rightarrow-a_{1}+i a_{2}=\alpha_{2}
\end{aligned}
$$

and calculating the partial derivatives as follows

$$
\begin{aligned}
& \frac{\partial \phi}{\partial u^{0}}=a_{0} \phi \\
& \frac{\partial^{2} \phi}{\partial u^{1^{2}}}=\frac{\partial}{\partial u^{1}}\left(\frac{\partial \phi}{\partial u^{1}}\right)=\frac{\partial}{\partial u^{1}}\left(a_{1} \phi\right)=a_{1} \frac{\partial \phi}{\partial u^{1}}=\left(a_{1}\right)^{2} \phi \\
& \frac{\partial^{2} \phi}{\partial u^{2}}=\frac{\partial}{\partial u^{2}}\left(\frac{\partial \phi}{\partial u^{2}}\right)=\frac{\partial}{\partial u^{2}}\left(a_{2} \phi\right)=a_{2} \frac{\partial \phi}{\partial u^{2}}=\left(a_{2}\right)^{2} \phi
\end{aligned}
$$

we verify the result obtained by equation 83 :

$$
a_{0}=\frac{i}{2 \mathcal{K}_{3} \hbar^{2}}\left(m_{0}^{2} c^{2}-\hbar^{2} \sum_{j=1}^{2} a_{j}^{2}\right)
$$

Since it is possible to write the coefficients $a_{1}, a_{2}$ and $a_{0}$, respectively, as

$$
\begin{aligned}
& a_{1}=\left(\frac{\alpha_{1}-\alpha_{2}}{2}\right) \\
& a_{2}=\left(\frac{\alpha_{1}+\alpha_{2}}{2 i}\right)
\end{aligned}
$$




$$
a_{0}=-\frac{i \alpha_{1} \alpha_{2}}{2 \mathcal{K}_{3}}+\frac{i m_{0}^{2} c^{2}}{2 \mathcal{K}_{3} \hbar^{2}}
$$

we can write the function $\Psi\left(u^{0}, u^{1}, u^{2}, u^{3}\right)$ in the form

$$
\Psi\left(u^{0}, u^{1}, u^{2}, u^{3}\right)=\exp \left(a_{0} u^{0}\right) \exp \left(a_{1} u^{1}\right) \exp \left(a_{2} u^{2}\right) \exp \left(a_{3} u^{3}\right)
$$

being $a_{3}=-i \mathcal{K}_{3}$. In next, applying the operator $\widetilde{\mathcal{H}}$ over the function $\Psi$, we obtain:

$$
\begin{aligned}
\widetilde{\mathcal{H}} \Psi\left(u^{0}, u^{1}, u^{2}, u^{3}\right) & =\widetilde{\widetilde{\mathcal{H}}}\left[\exp \left(-i \mathcal{K}_{3} u^{3}\right) \phi\left(u^{0}, u^{1}, u^{2}\right)\right] \\
& =\frac{i}{2 \mathcal{K}_{3} \hbar^{2}}\left((i \hbar)^{2} \sum_{j=1}^{2} a_{j}^{2}+m_{0}^{2} c^{2}\right) \Psi\left(u^{0}, u^{1}, u^{2}\right)
\end{aligned}
$$

In the meantime, we ought to calculate the operation of commutation between operators $\hat{a}_{1} \mathrm{e}$ $\hat{a}_{1}^{+}$as well as between operators $\hat{a}_{2}$ and $\hat{a}_{2}^{+}$with further application over the function $\phi\left(u^{0}, u^{1}, u^{2}\right)$. These calculations are shown in equations 89 and 90.

$$
\begin{aligned}
{\left[\hat{a}_{1}, \hat{a}_{1}^{+}\right]_{-} \phi } & =\hat{a}_{1} \hat{a}_{1}^{+} \phi-\hat{a}_{1}^{+} \hat{a}_{1} \phi \\
& =i\left(\frac{\partial^{2} \phi}{\partial u^{2} \partial u^{1}}-\frac{\partial^{2} \phi}{\partial u^{1} \partial u^{2}}\right)-i\left(\frac{\partial^{2} \phi}{\partial u^{2} \partial u^{1}}-\frac{\partial^{2} \phi}{\partial u^{1} \partial u^{2}}\right)+\sum_{j=1}^{2} \frac{\partial^{2} \phi}{\partial u^{j^{2}}}-\frac{\partial^{2} \phi}{\partial u^{j^{2}}} \\
& =0 \\
{\left[\hat{a}_{2}, \hat{a}_{2}^{+}\right]_{-} \phi } & =\hat{a}_{2} \hat{a}_{2}^{+} \phi-\hat{a}_{2}^{+} \hat{a}_{2} \phi \\
& =i\left(\frac{\partial^{2} \phi}{\partial u^{1} \partial u^{2}}-\frac{\partial^{2} \phi}{\partial u^{2} \partial u^{1}}\right)-i\left(\frac{\partial^{2} \phi}{\partial u^{1} \partial u^{2}}-\frac{\partial^{2} \phi}{\partial u^{2} \partial u^{1}}\right)+\sum_{j=1}^{2} \frac{\partial^{2} \phi}{\partial u^{j^{2}}}-\frac{\partial^{2} \phi}{\partial u^{j^{2}}} \\
& =0
\end{aligned}
$$

In turn, we can calculate the commutator between operators $\widetilde{\widetilde{\mathcal{H}}}$ and $\hat{a}_{1}$ with immediate application over the function $\phi\left(u^{0}, u^{1}, u^{2}\right)$, reaching the results presented by equations 91 and 92 .

$$
\begin{aligned}
& \widetilde{\mathcal{H}} \hat{a}_{1} \phi=-\frac{i}{2 \hbar}\left(i \hbar \frac{\partial}{\partial u^{3}}\right)^{-1}\left[m_{0}^{2} c^{2}+(i \hbar)^{2}\left(\sum_{j=1}^{2} \frac{\partial^{2}}{\partial u^{j^{2}}}\right)\right]\left(\frac{\partial \phi}{\partial u^{1}}+i \frac{\partial \phi}{\partial u^{2}}\right) \\
& =-\frac{i}{2 \hbar}\left(i \hbar \frac{\partial}{\partial u^{3}}\right)^{-1}\left[(i \hbar)^{2}\left(\frac{\partial^{3} \phi}{\partial u^{1^{3}}}+i \frac{\partial^{3} \phi}{\partial u^{1^{2}} \partial u^{2}}+\frac{\partial^{3} \phi}{\partial u^{2^{2}} \partial u^{1}}+i \frac{\partial^{3} \phi}{\partial u^{2^{3}}}\right)+\right. \\
& \left.m_{0}^{2} c^{2}\left(\frac{\partial \phi}{\partial u^{1}}+i \frac{\partial \phi}{\partial u^{2}}\right)\right] \\
& \hat{a}_{1} \widetilde{\mathcal{H}} \phi=\left(\frac{\partial}{\partial u^{1}}+i \frac{\partial}{\partial u^{2}}\right)\left[-\frac{i}{2 \hbar}\left(i \hbar \frac{\partial}{\partial u^{3}}\right)^{-1}\right]\left[(i \hbar)^{2}\left(\sum_{j=1}^{2} \frac{\partial^{2} \phi}{\partial u^{j^{2}}}\right)+m_{0}^{2} c^{2} \phi\right] \\
& =-\frac{i}{2 \hbar}\left[\left(i \hbar \frac{\partial}{\partial u^{3}}\right)^{-1}\right]\left[(i \hbar)^{2}\left(\frac{\partial^{3} \phi}{\partial u^{1^{3}}}+i \frac{\partial^{3} \phi}{\partial u^{1^{2}} \partial u^{2}}+\frac{\partial^{3} \phi}{\partial u^{2^{2}} \partial u^{1}}+i \frac{\partial^{3} \phi}{\partial u^{2^{3}}}\right)+\right. \\
& \left.m_{0}^{2} c^{2}\left(\frac{\partial \phi}{\partial u^{1}}+i \frac{\partial \phi}{\partial u^{2}}\right)\right]
\end{aligned}
$$




$$
\left[\widetilde{\mathcal{H}}, \hat{a}_{1}\right]_{-} \phi=\widetilde{\widetilde{\mathcal{H}}} \hat{a}_{1} \phi-\hat{a}_{1} \widetilde{\widetilde{\mathcal{H}}} \phi=0
$$

Furthermore, we calculate the operation of commutation between operators $\widetilde{\widetilde{\mathcal{H}}}$ and $\hat{a}_{1}^{+}$with immediate application on the function $\phi\left(u^{0}, u^{1}, u^{2}\right)$ in accordance with Equations 94 and 95.

$$
\begin{aligned}
\widetilde{\mathcal{H}} \hat{a}_{1}^{+} \phi & =-\frac{i}{2 \hbar}\left[\left(i \hbar \frac{\partial}{\partial u^{3}}\right)^{-1}\right]\left[(i \hbar)^{2}\left(\sum_{j=1}^{2} \frac{\partial^{2} \phi}{\partial u^{j^{2}}}\right)+m_{0}^{2} c^{2}\right]\left(\frac{\partial \phi}{\partial u^{1}}-i \frac{\partial \phi}{\partial u^{2}}\right) \\
& =-\frac{i}{2 \hbar}\left[\left(i \hbar \frac{\partial}{\partial u^{3}}\right)^{-1}\right]\left[(i \hbar)^{2}\left(\frac{\partial^{3} \phi}{\partial u^{1^{3}}}-i \frac{\partial^{3} \phi}{\partial u^{1} \partial u^{2}}+\frac{\partial^{3} \phi}{\partial u^{2} \partial u^{1}}-i \frac{\partial^{3} \phi}{\partial u^{2^{3}}}\right)+\right. \\
\hat{a}_{1}^{+} \widetilde{\mathcal{H}} \phi & \left.=-\frac{i}{2 \hbar}\left[\left(i \hbar \frac{\partial}{\partial u^{3}}\right)^{2}\right]\left(\frac{\partial \phi}{\partial u^{1}}-i \frac{\partial \phi}{\partial u^{2}}\right)\right] \\
& =-\frac{i}{2 \hbar}\left[\left(i \hbar \frac{\partial}{\partial u^{3}}\right)^{-1}\right]\left[(i \hbar)^{2}\left(\frac{\partial \phi}{\partial u^{2}}\right)\left[(i \hbar)^{2}\left(\sum_{j=1}^{2} \frac{\partial^{2} \phi}{\partial u^{1^{2}}}\right)+i \frac{\partial^{3} \phi}{\partial u^{1^{2}} \partial u^{2}}+\frac{m^{2} c^{2} \phi}{\partial u^{2} \partial u^{1}}-i \frac{\partial^{3} \phi}{\partial u^{2}}\right)+\right.
\end{aligned}
$$

$$
\left[\widetilde{\mathcal{H}}, \hat{a}_{1}^{+}\right]_{-} \phi=\widetilde{\widetilde{\mathcal{H}}} \hat{a}_{1}^{+} \phi-\hat{a}_{1}^{+} \widetilde{\widetilde{\mathcal{H}}} \phi=0
$$

Proceeding, similarly to the previous steps, we must calculate the commutator between operators $\widetilde{\mathcal{H}}$ and $\hat{a}_{2}$ with further application over function $\phi\left(u^{0}, u^{1}, u^{2}\right)$ :

$$
\begin{aligned}
& \widetilde{\mathcal{H}} \hat{a}_{2} \phi=-\frac{i}{2 \hbar}\left[\left(i \hbar \frac{\partial}{\partial u^{3}}\right)^{-1}\right]\left[(i \hbar)^{2}\left(\sum_{j=1}^{2} \frac{\partial^{2} \phi}{\partial u^{j^{2}}}\right)+m_{0}^{2} c^{2} \phi\right]\left(-\frac{\partial \phi}{\partial u^{1}}+i \frac{\partial \phi}{\partial u^{2}}\right) \\
&=-\frac{i}{2 \hbar}\left[\left(i \hbar \frac{\partial}{\partial u^{3}}\right)^{-1}\right]\left[(i \hbar)^{2}\left(-\frac{\partial^{3} \phi}{\partial u^{1^{3}}}+i \frac{\partial^{3} \phi}{\partial u^{1^{2}} \partial u^{2}}-\frac{\partial^{3} \phi}{\partial u^{2} \partial u^{1}}+i \frac{\partial^{3} \phi}{\partial u^{2}}\right)-\right. \\
&\left.\hat{a}_{2}{ }^{2} c^{2}\left(\frac{\partial \phi}{\partial u^{1}}-i \frac{\partial \phi}{\partial u^{2}}\right)\right]-\frac{i}{2 \hbar}\left[\left(i \hbar \frac{\partial}{\partial u^{3}}\right)^{-1}\right]\left(-\frac{\partial}{\partial u^{1}}+i \frac{\partial}{\partial u^{2}}\right)\left[(i \hbar)^{2}\left(\sum_{j=1}^{2} \frac{\partial^{2} \phi}{\partial u^{j^{2}}}\right)+m_{0}^{2} c^{2} \phi\right] \\
&=-\frac{i}{2 \hbar}\left[\left(i \hbar \frac{\partial}{\partial u^{3}}\right)^{-1}\right]\left[(i \hbar)^{2}\left(-\frac{\partial^{3} \phi}{\partial u^{1^{3}}}-i \frac{\partial^{3} \phi}{\partial u^{2} \partial u^{2}}+i \frac{\partial^{3} \phi}{\partial u^{2} \partial u^{1}}+i \frac{\partial^{3} \phi}{\partial u^{2}}\right)-\right. \\
&\left.m_{0}^{2} c^{2}\left(\frac{\partial \phi}{\partial u^{1}}-i \frac{\partial \phi}{\partial u^{2}}\right)\right]
\end{aligned}
$$

$\left[\widetilde{\mathcal{H}}, \hat{a}_{2}\right]_{-} \phi=\widetilde{\widetilde{\mathcal{H}}} \hat{a}_{2} \phi-\hat{a}_{2} \widetilde{\widetilde{\mathcal{H}}} \phi=0$ 
Ultimately, we proceed the calculate of commutator between operators $\widetilde{\mathcal{H}}$ and $\hat{a}_{2}^{+}$following with the application on function $\phi\left(u^{0}, u^{1}, u^{2}\right)$ :

$$
\begin{aligned}
& \widetilde{\widetilde{\mathcal{H}}} \hat{a}_{2}^{+} \phi=-\frac{i}{2 \hbar}\left[\left(i \hbar \frac{\partial}{\partial u^{3}}\right)^{-1}\right]\left[(i \hbar)^{2}\left(\sum_{j=1}^{2} \frac{\partial^{2} \phi}{\partial u^{j^{2}}}\right)+m_{0}^{2} c^{2} \phi\right]\left(-\frac{\partial \phi}{\partial u^{1}}-i \frac{\partial \phi}{\partial u^{2}}\right) \\
&=-\frac{i}{2 \hbar}\left[\left(i \hbar \frac{\partial}{\partial u^{3}}\right)^{-1}\right]\left[-(i \hbar)^{2}\left(\frac{\partial^{3} \phi}{\partial u^{1^{3}}}+i \frac{\partial^{3} \phi}{\partial u^{1} \partial u^{2}}+\frac{\partial^{3} \phi}{\partial u^{2} \partial u^{1}}+i \frac{\partial^{3} \phi}{\partial u^{2}}\right)-\right. \\
&\left.m_{0}^{2} c^{2}\left(\frac{\partial \phi}{\partial u^{1}}+i \frac{\partial \phi}{\partial u^{2}}\right)\right] \\
& \hat{a}_{2}^{+} \widetilde{\widetilde{\mathcal{H}}} \phi=-\frac{i}{2 \hbar}\left[\left(i \hbar \frac{\partial}{\partial u^{3}}\right)^{-1}\right]\left(\frac{\partial}{\partial u^{1}}-i \frac{\partial}{\partial u^{2}}\right)\left[(i \hbar)^{2}\left(\sum_{j=1}^{2} \frac{\partial^{2} \phi}{\partial u^{j^{2}}}\right)+m_{0}^{2} c^{2} \phi\right] \\
&=-\frac{i}{2 \hbar}\left[\left(i \hbar \frac{\partial}{\partial u^{3}}\right)^{-1}\right]\left[-(i \hbar)^{2}\left(\frac{\partial^{3} \phi}{\partial u^{1^{3}}}+i \frac{\partial^{3} \phi}{\partial u^{1^{2}} \partial u^{2}}+\frac{\partial^{3} \phi}{\partial u^{2} \partial u^{1}}+i \frac{\partial^{3} \phi}{\partial u^{2}}\right)-\right. \\
& {\left[\widetilde{\mathcal{H}}, \hat{a}_{2}^{+}\right]_{-} \phi }=\widetilde{\widetilde{\mathcal{H}}} \hat{a}_{2}^{+} \phi-\hat{a}_{2}^{+} \widetilde{\widetilde{\mathcal{H}}} \phi=0
\end{aligned}
$$

Based on the above expressions and taking advantage of Heisenberg formalism, we corroborate that the derivative with respect to time applied to the operator $\hat{a}_{i}$ (for $i=1,2$ ) and its adjunct operator $\hat{a}_{i}^{+}$is equal to zero in all cases, namely:

$$
\begin{aligned}
& -i \hbar \frac{d \hat{a}_{i}}{d t}=\left[\widehat{\mathcal{H}}, \hat{a}_{i}\right]_{-}=0, \quad i=1,2 \\
& -i \hbar \frac{d \hat{a}_{i}^{+}}{d t}=\left[\widehat{\mathcal{H}}, \hat{a}_{i}^{+}\right]_{-}=0, \quad i=1,2
\end{aligned}
$$

Therefore, we may assert that the operators $\hat{a}_{i}$ and their respective adjunct operators can be classified as operators of type integrals of motions.

\section{Conclusion}

The light cone coordinate system formalism has its appeal for facilitating the algebraic procedures and compression of the relativistic dynamics of physical systems, including notable applications in TQC and quantum computing.

In this sense, based on the approaches presented by Bagrov et al. (1976) and Kamassury et al. (2020), didactically develop the mathematical steps and physical considerations sufficient to construct the creation and annihilation operators so useful in studies of coherent quantum states (or quasi-classical states).

In addition to serving as a preparatory text for advanced studies in areas of contemporary physics, the pedagogical and sequential character of the presented approach, despite being oriented to the Klein-Gordon-Fock equation model for a free quantum particle, can be easily transported to other contexts and field settings. 


\section{References}

Bagrov, V. G., Buchbinder, I. L., \& Gitman, D. M. (1976). Coherent states of a relativistic particle in an external electromagnetic field. Journal of Physics A: Mathematical and General, 9(11), 1955-1965. doi: https://doi.org/10.1088/0305-4470/9/11/021

Bagrov, V. G., Gitman, D. M., \& Pereira, A. S. (2015). Coherent states of systems with quadratic hamiltonians. Brazilian Journal of Physics, 45, 369-375. doi: https://doi.org/10.1007/s13538$\underline{015-0309-\mathrm{Z}}$

Bagrov, V. G. \& Gitman, D. M. (1990). Exact solutions to relativistic wave equations. Springer.

Dirac, P. A. M. (1949). Forms of relativistic dynamics. Reviews of Modern Physics, 21(3), 392399. doi: https://doi.org/10.1103/RevModPhys.21.392

Gazeau, J. P. (2009). Coherent states in quantum physics. Wiley-VCH.

Kamassury, J. K. S., Meira Filho, D. P., Farias, S. A. S., \& Paraski, N. V. (2020). Obtenção da equação de Klein-Gordon-Fock em coordenadas do cone de luz. In Anais do XIV Simpósio Brasileiro de Engenharia Física (p.70). Santarém, PA, Brasil. doi: https://doi.org/10.22533/at.ed.1572002038

Meira Filho, D. P., \& Kamassury, J. K. S. (2018). Potência irradiada por uma carga elétrica acelerada no espaço-tempo de Minkowski. Revista Brasileira de Ensino de Física, 40(3), e3316-1e3316-19. doi: https://doi.org/10.1590/1806-9126-RBEF-2017-0398

Ohlsson, T. (2011). Relativistic quantum physics. Cambridge University Press.

Pereira, R. G., \& Miranda, E. (2002). Introdução à teoria quântica de campos: do oscilador harmônico ao campo escalar livre. Revista Brasileira de Ensino de Física, 24(2), 237-247. doi: https://doi.org/10.1590/S1806-11172002000200021

Stewart, J. (2012). Calculus. Cengage Learning.

Veeresha, P., Prakasha, D. G., \& Kumar, D. (2020). An eficiente technique for nonlinear timefractional Klein-Fock-Gordon equation. Applied Mathematics and Computation, 364, 124637-1-124637-15. doi: https://doi.org/10.1016/j.amc.2019.124637 